\title{
Approximating an Artificial General Intelligence or a General Collective Intelligence
}

Andy E. Williams

Nobeah Foundation, Nairobi, Kenya

\begin{abstract}
Human-Centric Functional Modeling (HCFM) has recently been used to define a model of Artificial General Intelligence (AGI) believed to have the capacity for human-like general problem-solving ability (intelligence), as well as a model of General Collective Intelligence (GCI) with the potential to combine individuals into a single collective intelligence that might have exponentially greater general problem-solving ability than any individual in the group. Functional modeling decouples the components of complex systems like cognition through well-defined interfaces so that they can be implemented separately, thereby breaking down the complex problem of implementing such a system into a number of much simpler problems. This paper explores how a rudimentary AGI and a rudimentary GCI might be implemented through approximating the functions of each, in order to create systems that provide sufficient value to incentivize more sophisticated implementations to be developed over time.
\end{abstract}

\section{Introduction}

This paper refers to what to the authors knowledge is the only model of Artificial General Intelligence (AGI) [1] with the potential to represent all the functions of human cognition, and what to the author's knowledge is the only model that defines the specific mechanisms required for a General Collective Intelligence or GCI with the potential for exponentially greater general problem-solving ability (intelligence) than any individual [2]. Because these models are the author's own work, and because these models are so new, all of the existing literature surrounding GCI refers to the author's own published work, or pre-prints of the author's unpublished work that is still under review. Therefore all the references cited in this paper are the authors own work as well.

\section{A Functional Model of Cognition and Collective Cognition}

The actions of any dynamical system with repeatable behavior can potentially be represented by the minimal set of functions with which that behavior can be composed. The states accessible through these functions then forms a "functional state space" through which the system moves. In the case of the cognitive system, a minimal set of functions has been defined with which it is believed to be possible to represent any reasoning processes (actively directed) or understanding processes (passively directed) with which the cognitive system might navigate through the space of concepts or "conceptual space" that serves as the functional state space of the mind. 


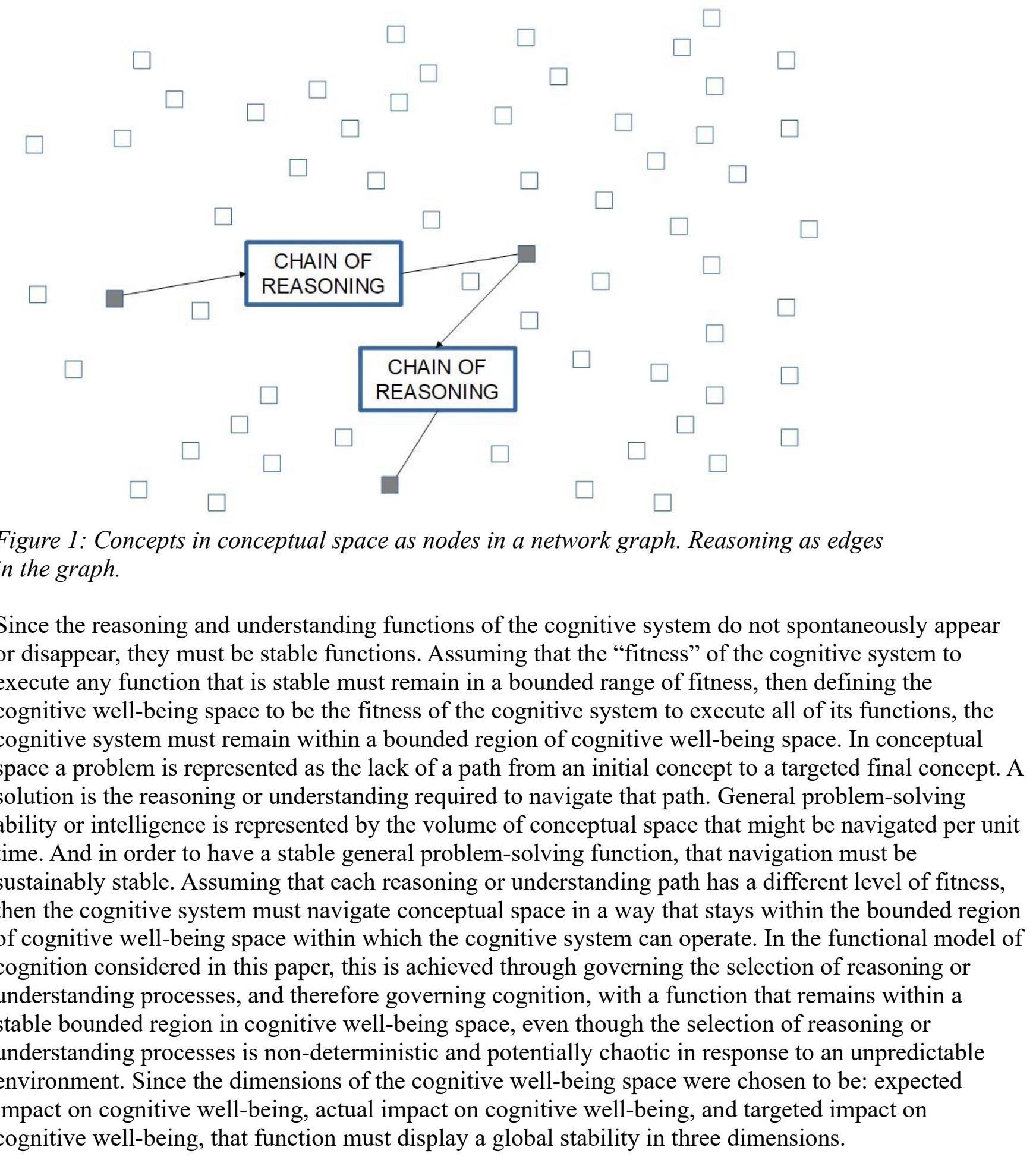




\section{Convection in the Cognitive Well- Being Space of the Conceptual}

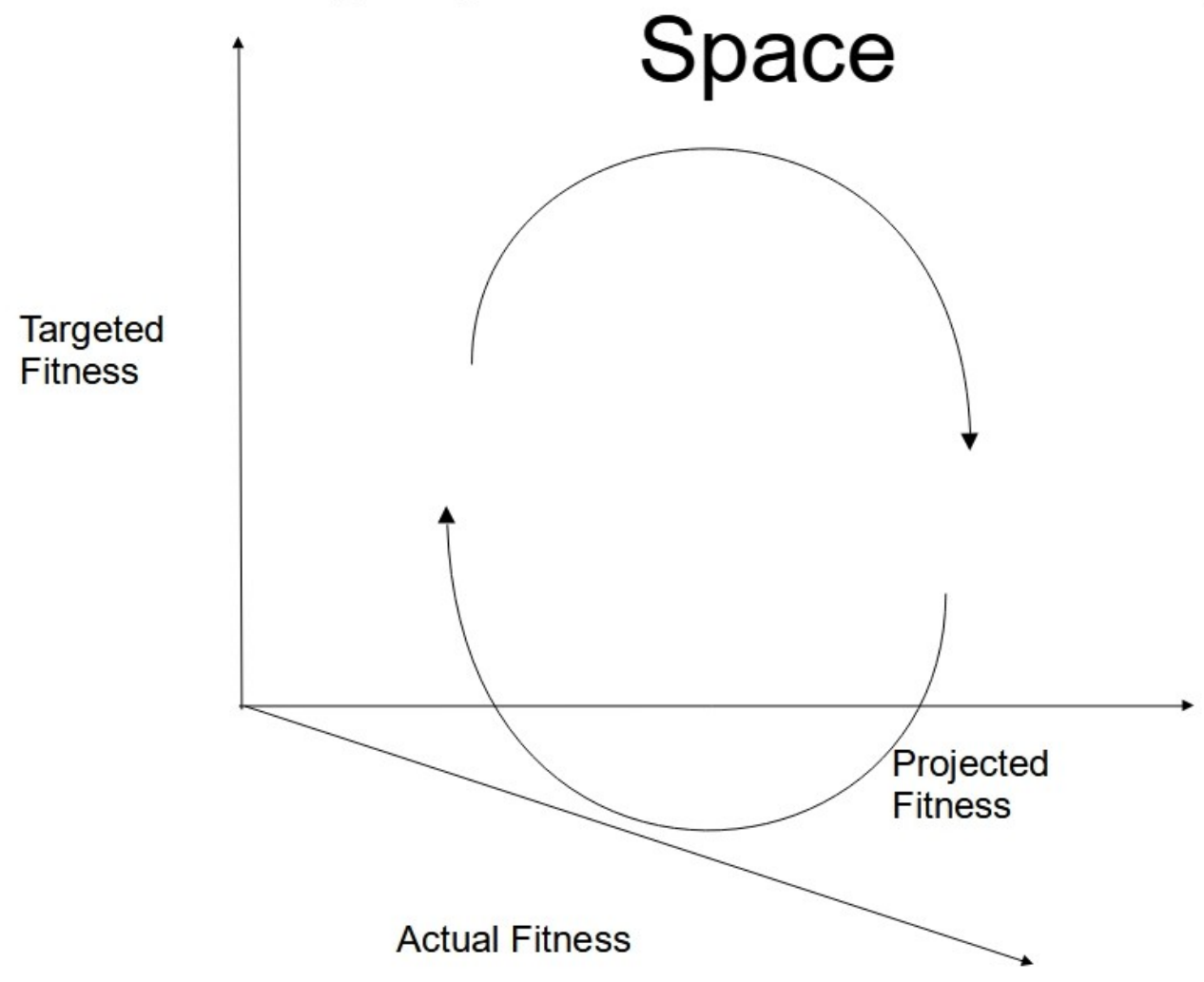

Figure 2: Stability in the operation of this model of the cognitive system is designed to be achieved through a set of equations which demonstrate that stability

One set of equations that provide this stability is the Lorenz equations for convection. By defining forces in fitness space (cognitive well-being space) to match these equations, and by defining the fitness of each reasoning or understanding process to navigate each path (i.e. to solve each problem) it is theorized that selection of reasoning and understanding might be sustainably stable. By incorporating all the functions believed to be required to orchestrate an unbounded number of functional components to execute this reasoning, then since this reasoning spans all reasoning possible, it is theorized that this model has the capacity to represent all functions of human cognition.

Component of Model

Functional modeling (problems and solutions) Functional decomposition

Functional domain bridging

\section{Description}

Components are modeled only by function to remove prejudice for or against any given implementation.

Functional components are decomposed into their most basic functional building blocks for reuse.

Different domains in which different functions are more fit in achieving the same purpose are identified. These domains are bridged by using a set of weights which identify the best function in each domain. 
Functional fitness

Functional stability

Functional adaptation
Every functional component is assigned some projected and actual fitness in achieving its function.

For functional components to persist they must display some degree of stability in fitness to function.

For functional components to persist in a changing environment they must have the ability to adapt their function.

Table 1: Components of process execution in functional model of cognition.

Because it is a functional model independent of implementation, it can potentially be implemented in a system of artificial cognition (an Artificial General Intelligence or AGI).

In the case of groups, by defining a collective conceptual space, collective reasoning or understanding processes can be defined as paths through that space in the same way. Where the functional components of an individual cognition might collaborate to execute reasoning, individuals might cooperate to execute reasoning in a system of collective cognition (a General Collective Intelligence or GCI) [3], [4]. Through defining a software platform that governs the selection processes in the same way, such a platform might combine groups into a single coherent reasoning process.

In either a system of individual or a system of collective cognition, since general problem-solving ability is defined by capacity to navigate conceptual space, that ability might be increased by increasing the complexity (length) of paths that can be navigated, by increasing the number of paths that can be navigated, and by increasing the capacity to sustain that navigation. Again, each path is a reasoning or understanding process. As the ability to generalize increases to a certain threshold, conceptual space can be demonstrated to undergo a phase change in which it's size and density increases exponentially, creating the potential to exponentially increase general problem-solving ability (intelligence) as well.

\section{Approximating Conceptual Space}

The conceptual space potentially allows the cognitive system to navigate from any concept to any other concept through any existing relationship between the two. Definitions of concepts in the conceptual space are fully self-contained, or in other words the conceptual space provides a complete representation of meaning. Since conceptual space is essentially a network graph, where concepts are the nodes in the graph, and in which relationships between concepts are the edges, that meaning is likely to be represented by characteristic patterns in that space. 


\section{Conceptual Depiction of Meaning Being Represented by a Pattern}

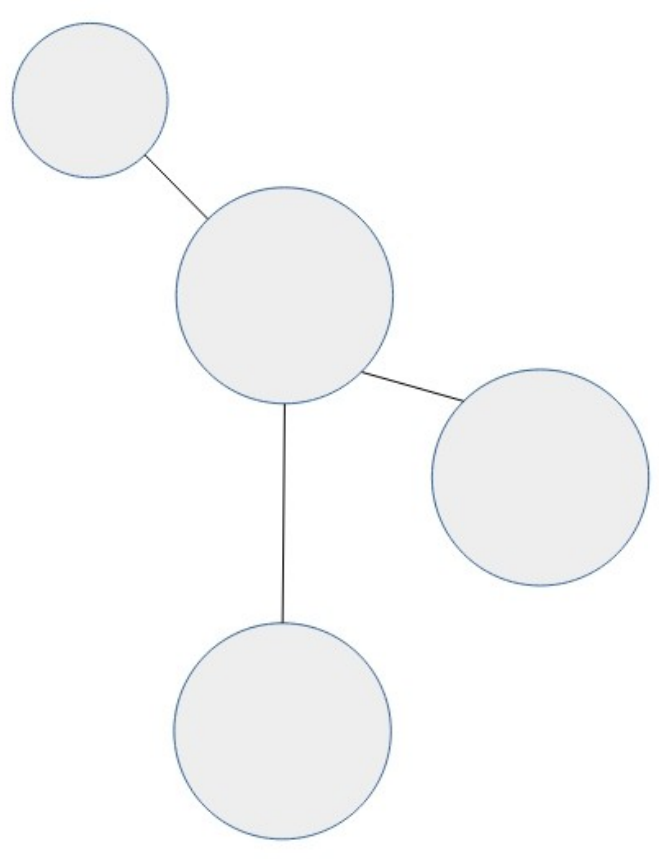

Dog

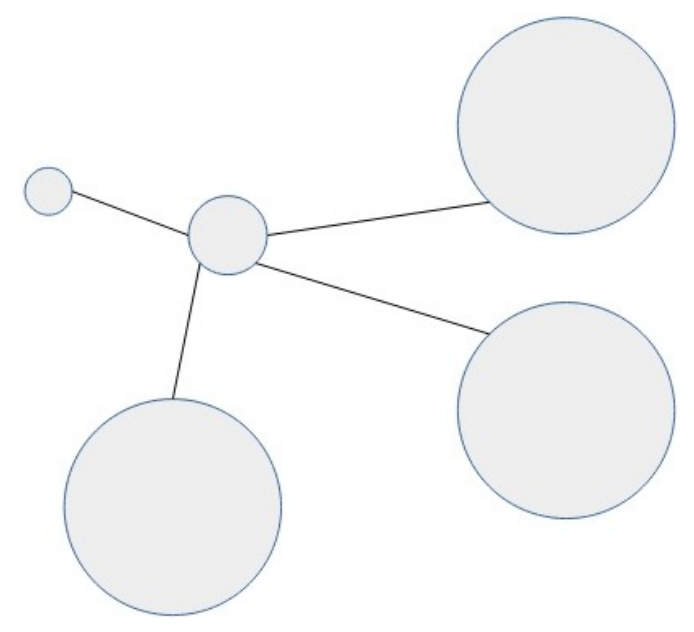

Cat

Figure 3: Different meanings might potentially be represented by different patterns. The pattern of a dog and the pattern of a cat might are not suggested to be represented by these exact patterns.

The conceptual space is then also a complete semantic representation of information that might permit any reasoning or understanding on any concept. The conceptual space has not yet been implemented however.

In the case of GCI, instead of using collective reasoning processes to navigate a collective conceptual space, it is possible to define a canonical (universally agreed upon) data model that all collective reasoning might be confined to. This might equate to defining each concept in terms of a fixed ontology. In a domain such as health care, for example, this might mean defining the concept "health" in terms of blood pressure, temperature, and heart rate. In a domain such as education, this might mean defining literacy in terms of a few key metrics. 


\section{Using a Canonical Data Model in Health Care}

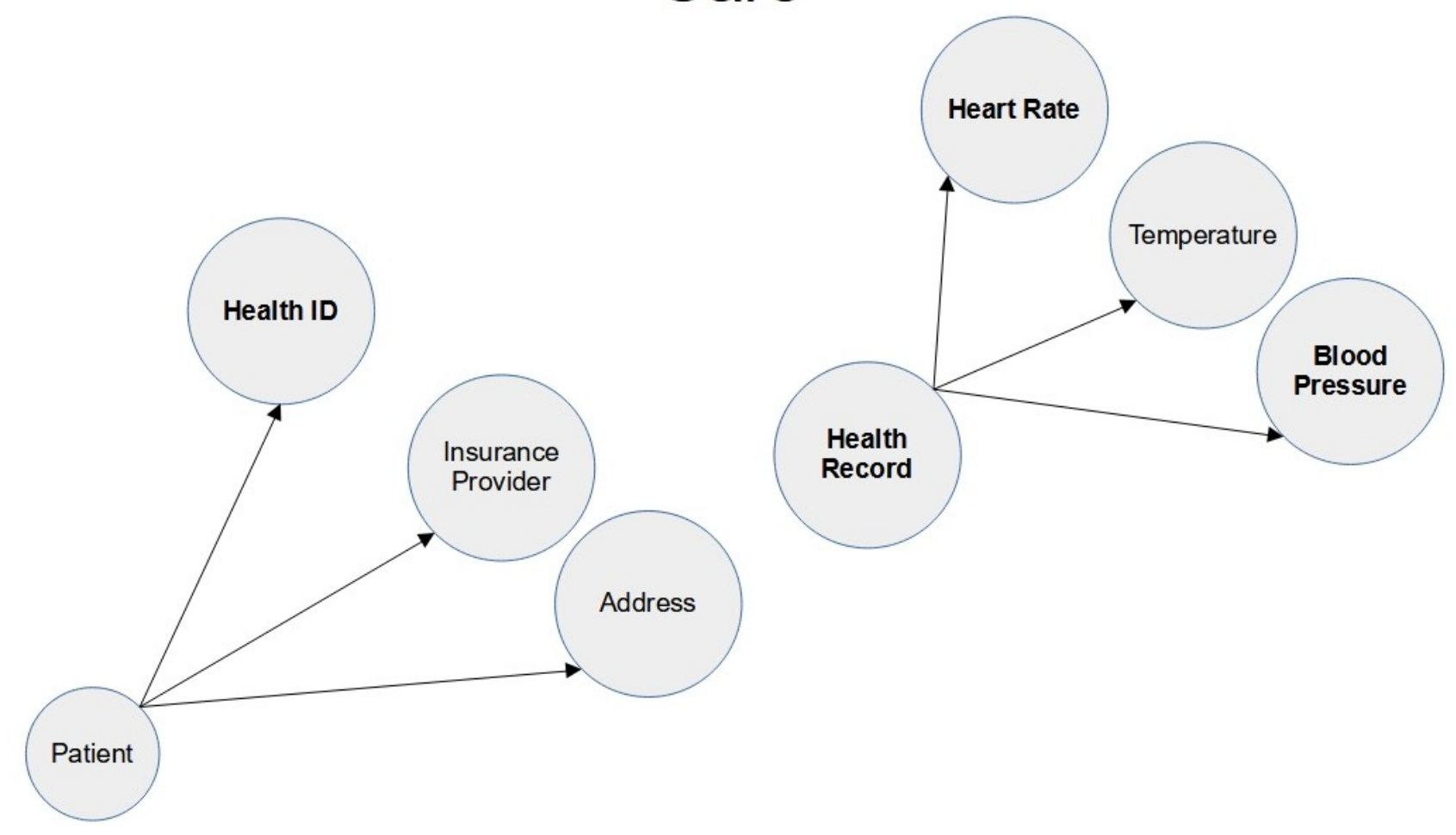

Figure 4: Examples of data elements in a canonical data model defined within the health care space.

In essence, rather than navigating the entire conceptual space, navigation is confined to the subset of the conceptual space represented by the ontology. And by restricting reasoning to that subset, the conceptual space can be implicitly used without needing to actually be implemented. 


\section{Canonical Data Model Implements Subset of Conceptual Space}

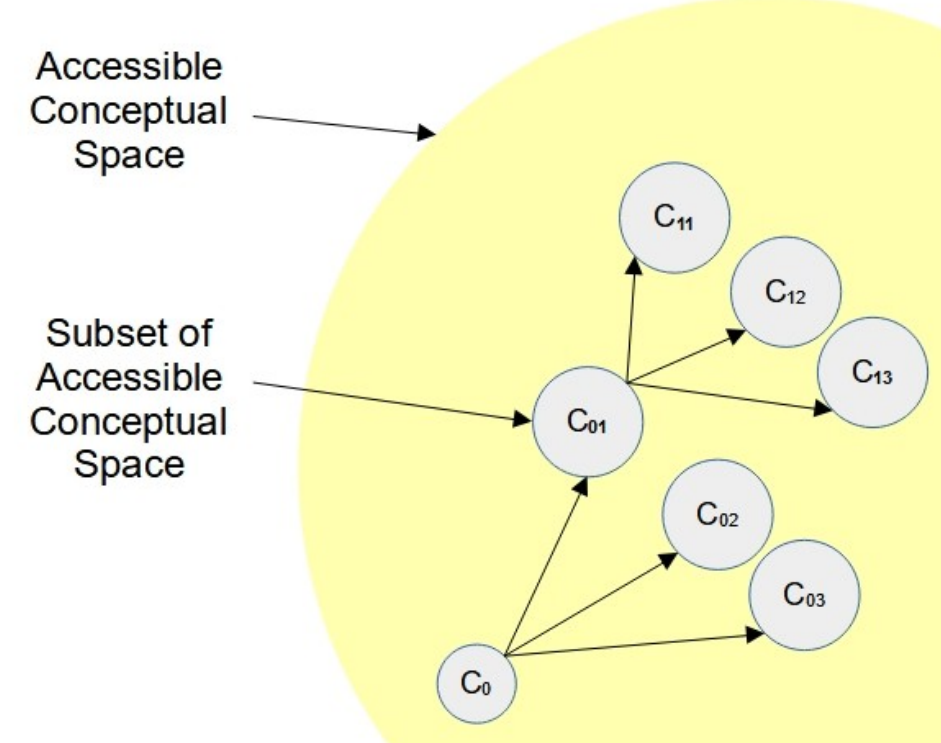

Figure 5: All the concepts in the canonical data model fall within the user's conceptual space and are therefore a subset of conceptual space.

\section{Approximating the Ability to Generalize}

In conceptual space a more general concept is a larger node that might enclose more specific (smaller) concepts. As the ability of the cognitive system to generalize increases, a node can potentially encompass all of conceptual space. As mentioned, solutions in conceptual space are paths between an initial concept, and a targeted final concept. Either of these concepts might be generalizations. As the ability to generalize reaches the point at which a generalization can encompass all of conceptual space, the number of potential reasoning paths (the number of edges in the graph representing the conceptual space) increases exponentially with the number of concepts in that space. Since the precision with which concepts can be located in conceptual space (their resolution) is defined by the number of reasoning paths fixing the location of each concept (the number of edges connected to each node), the density of that conceptual space might also increase significantly at this phase change. The ability to generalize is then required in order to exponentially increase intelligence through GCI.

However, since the conceptual space has not been implemented. This increase in intelligence must be achieved by approximating this generalization. One way is by manually generalizing all collective processes so they can potentially be reused in any other domain where they apply. One example is the process of forming cooperation. Assume that simple cooperation between two parties can be 
represented by a first order barter transaction in which one entity exchanges some value in return for the other party exchanging an approximately equal value. And assume that complex cooperation can be represented by Nth order barter transactions in which the first party gives something to the second party, who gives something to the third party, and so on until the $\mathrm{N}-1$ th party gives something to the Nth party. Nth order barter transactions can enable a GCI to optimize collective outcomes over an unlimited number of possible chains of cooperation, to the capacity of the cooperating entities to engage in that cooperation.

\section{Approximating the Forces in Cognitive Fitness Space}

The cognitive fitness space has also not yet been implemented, therefore it is not yet possible to define artificial forces in that space that will enable groups to converge on a single line of reasoning in a stable way. The general problem-solving ability of groups has been suggested to be measured by a general collective intelligence factor c [4]. While natural forces in groups might align collective reasoning to create an innate general collective intelligence factor [5], artificially achieving an exponential increase in that general collective intelligence factor through GCI requires implementing those forces explicitly.

However, these forces might be approximated through finding algorithms that improve the fitness of group decision-making in each specific case. As an example, take the case in which $\mathrm{N}$ potential participates in cooperation must select between $\mathrm{M}$ processes of cooperation, where $\mathrm{M}$ is greater than $\mathrm{N}$. The forces in collective well-being space are intended to maximize collective well-being. However, centralizing the decision of which chain of cooperation will be used so that it is made by any single individual might align the decision with that individual's interests, rather than with maximizing collective well-being. Such centralization would then represent individuals reasoning individually within a group, rather than representing collective reasoning. One potential collective reasoning process is for the first individual to select the top N-1 chains of cooperation from their perspective, the second individual to select the top N-2 chains of cooperation from their perspective, and so forth until the Nth individual selects the final chain of cooperation that all $\mathrm{N}$ actors participate to execute.

\section{An Example}

Rather than funding individual startup businesses which in general might be ninety percent likely to fail, a bank or investor might fund an entire value chain of businesses based on collectively intelligent cooperation in which one business agrees to buy a key product or service from another business in the chain, in return for another business in the chain agreeing to buy a key product or service from them [6]. Assume one chain of cooperation involves a distributor of school uniforms who buys the uniforms from a manufacturer, a not-for-profit that trains the seamstresses, a manufacturer who hires the seamstresses and buys cloth from a weaver, and a weaver who buys cotton from a grower. This might be one of several chains of cooperation that might be funded by the bank, that might be supported by the NGO, and that might be engaged in by local entrepreneurs. Rather than the current model in which the bank essentially selects which business is funded, a platform might be created that allows all banks to cooperate in selecting the best value chains according to their criteria (e.g. high ROI and low risk), that allows all NGOs to cooperate in selecting the subset of value chains selected by the banks that are the best according to their criteria (e.g. high number of jobs created per funds donated), and that allows local entrepreneurs to select the actual value chain that will be implemented according to what is best to them (e.g. highest livelihood). The most fit bank, the most fit NGO, and the most fit entrepreneurs might then be selected by the platform to implement that value chain. This cooperation ensures sales for each business even before it starts, thereby significantly reducing risk for both the businesses as well as for the lenders or investors. This reduction in risk together with cooperation with the NGO can significantly increase returns as well. Therefore through such cooperation, all participants might significantly increase their individual outcomes, thereby increasing collective outcomes. 


\section{Implications}

General processes have been conceptualized to use the conceptual space as a method for individually customizing any product, such as a renewable energy solution, or any service, such as education or health care, in order to maximize collective outcomes through maximizing outcomes for each individual. And processes of generalization have been defined in that conceptual space so that an adaptive problem-solving system such as an AGI or a GCI might achieve exponentially increased general problem-solving ability through executing those generalizations on both concepts and reasoning. Though those processes cannot be executed in conceptual space because conceptual space has not yet been implemented, and though a complete AGI or GCI cannot be executed since the fitness space has not yet been implemented approximations for this functionality might be used to essentially implement a subset of AGI or GCI functionality, and in doing so to gain a significant increase in outcomes.

A proposed Collective Intelligence based Program to Accelerate Achievement of the Sustainable Development Goals (CIPAA-SDGs) incorporates this approximation approach. In phase I of this program which focuses on agricultural livelihoods, this approximation approach has been used to define generalized chains of cooperation that create the potential to increase impact per government or donor program dollar by up to $750 \mathrm{X}$ [6]. In phase II of this program, which focuses on education, health care, renewable energy and other areas, application of this approximation approach to health care platforms creates the potential to significantly increase access to affordable health care [7]. And application of this approach to education platforms creates the potential to significantly increase access to affordable education, or to improve the educational outcomes that can be achieved at a given cost [8].

\section{Conclusions}

Although much work remains to implement all the functional components of an AGI or a GCI, by making some simple approximations, very significant value can be derived in order to incentivize investment in elaborating this functional model and its implementation.

\section{References}

[1] Williams A.E. (2020) A Model for Artificial General Intelligence. In: Goertzel B., Panov A., Potapov A., Yampolskiy R. (eds) Artificial General Intelligence. AGI 2020. Lecture Notes in Computer Science, vol 12177. Springer, Cham. https://doi.org/10.1007/978-3-030-52152-3_38

[2] Williams, A. E. (2020, April 30). A Model for General Collective Intelligence. https://doi.org/10.31730/osf.io/6u984

[3] The Relationship Between Collective Intelligence and One Model of General Collective Intelligence, Andy E. Williams, Computational Collective Intelligence, 11th International Conference, ICCCI 2019, Hendaye, France, September 4-6, 2019, Proceedings, Part II, Pages 589-600 [4] Williams, A. E. (2020, December 2). General Collective Intelligence vs the Innate Collective Intelligence Factor. https://doi.org/10.31730/osf.io/kp3x8

[5] Woolley, Anita Williams; Chabris, Christopher F.; Pentland, Alex; Hashmi, Nada; Malone, Thomas W. (29 October 2010). "Evidence for a Collective Intelligence Factor in the Performance of Human Groups". Science. 330 (6004): 686-688. Bibcode:2010Sci...330..686W. doi:10.1126/science.1193147. PMID 20929725. S2CID 74579.

[6] Williams, A. E. (2020, April 30). The Collective Intelligence based Program to Accelerate Achievement of the Sustainable Development Goals as a Case Study for Collectively Intelligent Program Design. https://doi.org/10.31235/osf.io/r2dxq 
[7] Williams, A. E. (n.d.). Functional Modeling and General Collective Intelligence as the Basis for Pervasive Healthcare. Retrieved from osf.io/preprints/africarxiv/4wzft

[8] Williams, A. E. (n.d.). The Role of General Collective Intelligence in Optimizing Future Educational Environments. Retrieved from osf.io/preprints/africarxiv/ewpur 